\title{
Macroscopic theory of giant magnetoresistance in magnetic granular metals
}

\author{
R. Y. Gu and L. Sheng \\ Department of Physics and National Laboratory of Solid State Microstructures, Nanjing University, Nanjing 210093, \\ People's Republic of China \\ D. Y. Xing \\ Chinese Center of Advanced Science and Technology (World Laboratory), P.O. Box 8730, Beijing, People's Republic of China \\ and Department of Physics, Nanjing University, Nanjing 210093, People's Republic of China \\ Z. D. Wang \\ Department of Physics, University of Hong Kong, Pokfulam Road, Hong Kong \\ J. M. Dong \\ Department of Physics and National Laboratory of Solid State Microstructures, Nanjing University, Nanjing 210093, \\ People's Republic of China
}

(Received 29 June 1995; revised manuscript received 28 September 1995)

\begin{abstract}
A macroscopic theory of giant magnetoresistance in granular magnetic materials is developed to improve on that of Rubinstein [Phys. Rev. B 50, 3830 (1994)]. By using a self-consistent method and introducing a useful parametrization, we show the magnetotransport in granular systems to be between those for currents in the plane of layers and currents perpendicular to the plane of the layers in multilayers. The theoretical result in the local limit is found to be in agreement with the observed singular dependence of the giant magnetoresistance on annealing temperature.
\end{abstract}

\section{INTRODUCTION}

The discovery of giant magnetoresistance (GMR) effects in a number of antiferromagnetically coupled multilayers such as $\mathrm{Fe} / \mathrm{Cr}$ and $\mathrm{Co} / \mathrm{Cu}$ has attracted much attention in experimental and theoretical study. ${ }^{1-4}$ A large negative magnetoresistance was found first for currents in the plane of the layers (CIP) (Refs. 1,2) and subsequently for currents perpendicular to the plane of the layers (CPP). ${ }^{3,4}$ Recently, a similar GMR effect has been observed in heterogeneous (granular) $\mathrm{Co}-\mathrm{Cu}$ (Ref. 5) and Co-Ag films, ${ }^{6}$ composed of isolated ferromagnetic granules embedded in a nonmagnetic matrix.

On the theoretical side, to account for the CIP-MR, two different transport theories have been developed: a quasiclassical method based on the Boltzmann equation ${ }^{7-10}$ and a quantum approach starting from Kubo formula. ${ }^{11,12}$ Both of them attribute the GMR effect to the spin-dependent scattering at ferromagnetic-nonmagnetic interfaces and within ferromagnetic layers. Their common physical content is that the electrons average the properties of the multilayers in the perpendicular direction on the length scale of the electron mean free path (MFP). This implies that the GMR vanishes when the repetition length $d$ of the layered pattern becomes larger than the MFP $\lambda .^{7,11}$ For the CPP geometry, ${ }^{13-15}$ spin accumulation effects must be taken into account so that the actual electric field is no longer uniform as it is in the CIP geometry. The total electric field is the sum of the external field and the internal field produced by the charge accumulation; the latter depends not only on the coordinate perpendicular to the plane, but also on the spin of the conduction electrons. In general, owing to existence of spin-flip (spin mixing), the current for each spin is not constant. In the case appropriate for most experiments, however, $d$ is much shorter than the spin-diffusion length $l_{\text {sf }}$ so that the spin-flip scattering can be neglected, ${ }^{14,15}$ and the current for each spin remains constant. In particular, in the local limit $\lambda \ll d$ (but still $d$ is much smaller than $l_{\text {sf }}$ ), the CPP (CIP) geometry can simply reduce to a resistor network where the resistances of individual layers for each spin direction are added in series (parallel), while those for the two spin channels are added in parallel. ${ }^{14,15}$ Under this limit, the CPP-MR does exist while the CIP-MR vanishes. On the other hand, the theories of the MR in granular systems have not been quite well developed as the spatial distributions of the fields and the currents are more complicated in these inhomogeneous systems. Spin dependent scattering at the interfaces between granules and the matrix and within the granules is still believed to be the main mechanism of GMR in granular systems. ${ }^{5,16-18}$ It was proposed that the transport in granular films is very close to the CPP case in multilayers, ${ }^{16,17}$ which is equivalent to an assumption of a uniformly distributed current density. Such theories predict a monotonously decreasing GMR with increasing the particle sizes, if the applied magnetic field is strong enough to saturate the global magnetization of the systems. The observed maximum of the GMR at a certain size was considered to stem from the superferromagnetic effect that the smaller the particle size becomes, the more difficult to saturate the global magnetization. ${ }^{17}$ Recently, Rubinstein ${ }^{18}$ made an attempt to extend the theory of Valet and Fert ${ }^{14}$ which deals with the CPP-MR of multilayers to the granular systems, but had great difficulty in accounting for experimental results of GMR. Zhao and $\mathrm{Pu}^{19}$ gave a quantum treatment for the GMR in granular systems by vir- 
tue of an autocorrelation-function description of interfacial roughness. Camblong, Levy, and Zhang ${ }^{20}$ developed a theory of electron transport in inhomogeneous magnetic structures that are oriented noncollinearly, in which it is necessary to introduce currents, fields, and conductivity tensors that are off diagonal in the spin space of the conduction electrons.

In this paper we develop a macroscopic theory of GMR in granular magnetic materials to improve on that of Ref. 18. First, we use an effective conductivity, defined as the average current density over the average internal electric field, in lieu of the measurable conductivity. Second, we consider finite conductivities in both spin channels. Third, an improved self-consistency is developed for the calculation of averages. The spin-dependent scattering both at interfaces and within ferromagnetic granules are taken into account. The interfaces between the ferromagnetic granules and the nonmagnetic matrix are treated as thin regions of mixed ferromagnetic and nonmagnetic atoms. Such a treatment was first proposed by Johnson and Camley ${ }^{8}$ to account simultaneously for both the CIP-MR and the overall CIP resistivity of magnetic multilayers. In this model the bulk and interfacial scattering are treated in the same way because the spindependent scattering at the sharp interfaces has been replaced by the scattering within the mixed regions. As same as most GMR theories, we limit our theory to the case where $l_{\mathrm{sf}}$ is much larger than both the MFP and the size of ferromagnetic regions so that the spin-flip effect can be neglected and the two-current model is applicable. It has been shown that this condition is satisfied for most experiments, as discussed in Appendix A of Ref. 14. To compare the magnetotransport in granular systems with those for the CIP and CPP geometry in multilayers, by introducing a useful parametrization, we show that at least in the local limit $(\lambda \ll d$, where $d$ is the inhomogeneous length scale) the transport in granular systems is between those for the CPP and CIP cases in magnetic multilayers. Using the present theory, we have a discussion on the size dependence of the GMR in granular systems. The calculated results show that with a decrease in size of the ferromagnetic granules, the GMR first increases and then decreases, having a maximum in the middle region. This theoretical result is in agreement with the observed singular dependence of the GMR on annealing temperature. We attribute this feature of the GMR to a competition of two factors in determining the magnetotransport properties. One is that the proportion of the spin-dependent scattering in the total scattering increases with decreasing the size of the granules; the other is that the decrease in size can enhance the effect that the currents bypass the high-resistivity granules, leading to a crossover of the magnetotransport from CPP to CIP. To our knowledge, the latter factor has not yet been considered in the previous literature.

\section{MACROSCOPIC THEORY}

Let us consider a granular system consisting of ferromagnetic granules embedded in a nonmagnetic matrix. In the absence of the magnetic field the magnetization directions of the granules are random. ${ }^{20}$ However, in almost all theoretical works on GMR, except Ref. 20, only collinear magnetization configurations of the granules are considered, i.e., all the ferromagnetic granules are assumed to have only two magnetization directions: up $(\uparrow)$ and down $(\downarrow)$, with the quantization axis along the direction of the applied magnetic field. Such a simplifying treatment is an approximation of a magnetic granular system, which is similar to that of using the Ising model to describe a ferromagnetic system. In the present macroscopic theory we use this collinear approximation and expect the results obtained to be valid at least qualitatively.

It has been shown ${ }^{15}$ that in the local limit and in the absence of spin-flip scattering, the relation between the current density $\mathbf{J}_{s}(\mathbf{r})$ and the electric field $\mathbf{E}_{s}(\mathbf{r})$ at point $\mathbf{r}$ takes the local form of the Ohm's law

$$
\mathbf{J}_{s}(\mathbf{r})=\sigma_{s}(\mathbf{r}) \mathbf{E}_{s}(\mathbf{r}),
$$

where the sign $s=\uparrow(\downarrow)$ is the absolute spin direction, and the local conductivity $\sigma_{s}(\mathbf{r})$ can be written as

$$
\sigma_{s}(\mathbf{r})= \begin{cases}\sigma_{s i}^{f}, & \text { if } \mathbf{r} \text { is in the } i \text { th ferromagnetic granule, } \\ \sigma_{s i}^{m}, & \text { if } \mathbf{r} \text { is in the } i \text { th mixed region, } \\ \sigma_{n}, & \text { if } \mathbf{r} \text { is in the nonmagnetic matrix, }\end{cases}
$$

with $\sigma_{s i}^{f}\left(\sigma_{s i}^{m}\right)$ being $\sigma_{+}^{f}\left(\sigma_{+}^{m}\right)$ for spin $s$ parallel to the $i$ th granule's magnetization direction $\mathbf{M}_{\mathbf{i}}\left(s \| \mathbf{M}_{\mathbf{i}}\right)$ and $\sigma_{-}^{f}\left(\sigma_{-}^{m}\right)$ for spin $s$ antiparallel to $\mathbf{M}_{\mathbf{i}}\left(s \|-\mathbf{M}_{\mathbf{i}}\right)$. Throughout the paper, we use the sign $\alpha=+,-$, respectively, to denote the majority and minority spin directions (spin parallel and antiparallel to the local magnetization) in a magnetic granule. If we introduce $\tau_{\alpha}^{f}, \tau_{\alpha}^{m}$, and $\tau_{n}$ as the relaxation times in the granules, mixed regions, and matrix, respectively, we have

$$
\sigma_{\alpha}^{f}=\frac{n e^{2} \tau_{\alpha}^{f}}{m}
$$

$$
\begin{gathered}
\sigma_{\alpha}^{m}=\frac{n e^{2} \tau_{\alpha}^{m}}{m}, \\
\sigma_{n}=\frac{n e^{2} \tau_{n}}{m},
\end{gathered}
$$

where $e$ is the charge of an electron, $m$ is the effective mass of conduction electrons, and $n$ is the density of conduction electrons. Here we wish to point out that the conductivity in the mixed region must be very small, otherwise the conduc- 
tion electrons could not feel the existence of the interface scattering when they pass the mixing regions.

To show explicit results of conductivity and GMR in the local limit, we adopt a simplified picture of the structure of the granular alloys, similar to that used in Ref. 18. We assume that all the ferromagnetic granules are spherical in shape and have the same radius $a$, each thin mixed region being a spherical shell with a small thickness of $t$. Define $f$ as the volumetric filling factor of the ferromagnetic granules, and $N$ the number of granules of the system; then

$$
f=\frac{N V_{g}}{V},
$$

where $V_{g}=\frac{4}{3} \pi a^{3}$ is the volume of each granule and $V$ is the total volume of the system.

Since the average current density $\left\langle\mathbf{J}_{s}\right\rangle$ has the same direction as that of the average electric field $\left\langle\mathbf{E}_{s}\right\rangle$, the average conductivity for spin $s$ channel of a granular system is

$$
\sigma^{\sim}{ }_{s}=\frac{\left\langle\mathbf{J}_{s}\right\rangle}{\left\langle\mathbf{E}_{s}\right\rangle},
$$

with the average field and current density being

$$
\begin{aligned}
&\left\langle\mathbf{E}_{s}\right\rangle= \frac{1}{V}\left(\sum_{i} \int_{i \mathrm{th}} d^{3} r \mathbf{E}_{s}(\mathbf{r})+\sum_{i} \int_{i \text { th mixed }} d^{3} r \mathbf{E}_{s}(\mathbf{r})\right. \\
&\left.+\int_{\text {matrix }} d^{3} r \mathbf{E}_{s}(\mathbf{r})\right), \\
&\left\langle\mathbf{J}_{s}\right\rangle=\frac{1}{V}\left(\sum \int_{i \text { th }} d^{3} r \sigma_{s i}^{f} \mathbf{E}_{s}(\mathbf{r})+\int_{\text {matrix }} d^{3} r \sigma_{n} \mathbf{E}_{s}(\mathbf{r})\right),
\end{aligned}
$$

where the subscripts " $i$ th," "ith mixed," and "matrix" mean that the integral is performed in the $i$ th granule, the $i$ th mixed region, and the matrix, respectively, and the sum is over all the granules embedded in the matrix. In the second formula of Eq. (6) we have omitted the contribution of the mixed regions to the average current density, for the volumetric filling factor of these regions is very small and the conductivities in these regions are much smaller than in the granules and in the matrix. On the other hand, due to spin accumulation effect there are strong internal electric fields in the mixed regions. Their contribution to the average electric field should be taken into account. Here we wish to point out that a sum of the average conductivity $\widetilde{\sigma}_{\uparrow}$ and $\widetilde{\sigma}_{\downarrow}$ defined by Eq. (5) is very closed to the measurable conductivity. In the two-current model the measurable conductivity of a sample is the sum of $\sigma_{\uparrow}$ and $\sigma_{\downarrow}$ with $\sigma_{s}=\left(I_{s} / U\right)(L / S)$ where $L$ is the sample length along the current direction and $S$ is the area of cross section, $I_{\uparrow}+I_{\downarrow}$ is the measurable current through the sample, and $U$ is the potential drop at its terminals. Since $I_{s}=\left\langle\mathbf{J}_{s}\right\rangle S$, and $U=L\left\langle\mathbf{E}_{\uparrow}\right\rangle=L\left\langle\mathbf{E}_{\downarrow}\right\rangle$, we have $\sigma_{s}=\widetilde{\sigma}_{s}$, indicating that the use of the effective conductivities defined in Eq. (5) in lieu of the measurable conductivity is justifiable.

As shown in Eqs. (2) and (3), the local conductivity in a granule depends on the relative spin direction of conduction electrons in the granule. The electric field in the granule also depends on the relative spin direction, e.g., in the $i$ th granule, $\mathbf{E}_{\mathbf{s}}(\mathbf{r})$ is equal to $\mathbf{E}_{+}(\mathbf{r})$ for $s \| \mathbf{M}_{\mathbf{i}}$ and $\mathbf{E}_{-}(\mathbf{r})$ for $s \|-\mathbf{M}_{\mathbf{i}}$. It is convenient to define two kinds of average electric fields in all the ferromagnetic granules: $\overline{\mathbf{E}}_{\alpha}^{\prime<}$ and $\overline{\mathbf{E}}_{\alpha}^{<}$, corresponding to the average fields including and excluding the contribution of the mixed regions, respectively,

$$
\begin{gathered}
\overline{\mathbf{E}}_{\alpha}^{<}=\frac{1}{N V_{g}} \sum_{i} \int_{i t \mathrm{~h}} d^{3} r \mathbf{E}_{\alpha}(\mathbf{r}), \\
\overline{\mathbf{E}}_{\alpha}^{\prime<}=\mathbf{E}_{\alpha}^{<}+\frac{1}{N V_{g}} \sum_{i} \int_{i \text { th mixed }} d^{3} r \mathbf{E}_{\alpha}(\mathbf{r}),
\end{gathered}
$$

where the sum is over all the granules, and $\alpha=+(-)$ for majority (minority) spin direction. Similarly, the average field in the matrix $\overline{\mathbf{E}}_{\alpha}^{>}$is given by

$$
\overline{\mathbf{E}}_{+(-)}^{>}=\frac{1}{V-N V_{g}} \int_{\text {matrix }} d^{3} r \mathbf{E}_{\uparrow(\downarrow)}(\mathbf{r}) .
$$

Since MR is defined as the difference of resistivity between the completely magnetized state and the completely demagnetized state, in what follows we focus attention on these two states. For the magnetized state, all the granules have the same magnetization direction (pointed up); the average field and current density in Eq. (6) reduce to

$$
\begin{gathered}
\left\langle\mathbf{E}_{\uparrow(\downarrow)}\right\rangle=f{\overline{\mathbf{E}^{\prime}}}_{+(-)}^{<}+(1-f) \overline{\mathbf{E}}_{+(-)}^{>}, \\
\left\langle\mathbf{J}_{\uparrow(\downarrow)}\right\rangle=f \sigma_{+(-)}^{f} \overline{\mathbf{E}}_{+(-)}^{<}+(1-f) \sigma_{n} \overline{\mathbf{E}}_{+(-)}^{>} .
\end{gathered}
$$

According to Eq. (5) the average conductivities for spin-up and spin-down channels can be obtained, and the measured total conductivity is the sum of them,

$$
\sigma^{M}=\frac{f k_{+} \sigma_{+}^{\prime}+(1-f) \sigma_{n}}{f k_{+}+1-f}+\frac{f k_{-} \sigma_{-}^{\prime}+(1-f) \sigma_{n}}{f k_{-}+1-f},
$$

where $\sigma_{\alpha}^{\prime}=\bar{E}_{\alpha}^{<} \sigma_{\alpha}^{f} / \overline{E^{\prime}}>$ is the effective conductivity by taking the effect of the mixed regions into account, and $k_{\alpha}$ $=\overline{E^{\prime}}<{ }_{\alpha} / \bar{E}_{\alpha}^{>}$is an important parameter which will be discussed below.

In a demagnetized sample, there are equal numbers of granules with magnetization pointed up and down. Such a symmetry results in $\overline{\mathbf{E}}_{+}^{>}=\overline{\mathbf{E}}_{-}^{>}$, yielding

$$
\begin{gathered}
\left\langle\mathbf{E}_{\uparrow}\right\rangle=\left\langle\mathbf{E}_{\downarrow}\right\rangle=(f / 2)\left(\overline{\mathbf{E}^{\prime}}<+\overline{\mathbf{E}^{\prime}}<\right)+(1-f) \overline{\mathbf{E}}_{+}^{>}, \\
\left\langle\mathbf{J}_{\uparrow}\right\rangle=\left\langle\mathbf{J}_{\downarrow}\right\rangle=(f / 2)\left(\sigma_{+}^{f} \overline{\mathbf{E}}_{+}^{<}+\sigma_{-}^{f} \overline{\mathbf{E}}_{-}^{<}\right)+(1-f) \sigma_{n} \overline{\mathbf{E}}_{-}^{>},
\end{gathered}
$$

so that the conductivity of the demagnetized state is

$$
\sigma^{D}=2 \frac{(f / 2)\left(k_{+} \sigma_{+}^{\prime}+k_{-} \sigma_{-}^{\prime}\right)+(1-f) \sigma_{n}}{(f / 2)\left(k_{+}+k_{-}\right)+1-f},
$$

and then the MR of the system is obtained by $\mathrm{MR}=\left(\sigma^{M}-\sigma^{D}\right) / \sigma^{D}$.

Now let us see the physical meaning of $k_{\alpha}$. When $k_{\alpha}=1$, Eqs. (9) and (10) become

$$
\sigma^{M}=\sigma^{D}=f \sigma_{+}^{\prime}+f \sigma_{-}^{\prime}+2(1-f) \sigma_{n},
$$

which corresponds to a resistor network in parallel shown in Fig. 1. We know that in the local limit, the CIP resistances of a magnetic multilayer reduce to a resistor network in parallel and the CIP-MR vanishes. Thus $k_{\alpha}=1$ corresponds to the 


\section{$\stackrel{\mathbf{E}}{\overrightarrow{C P P}}$}

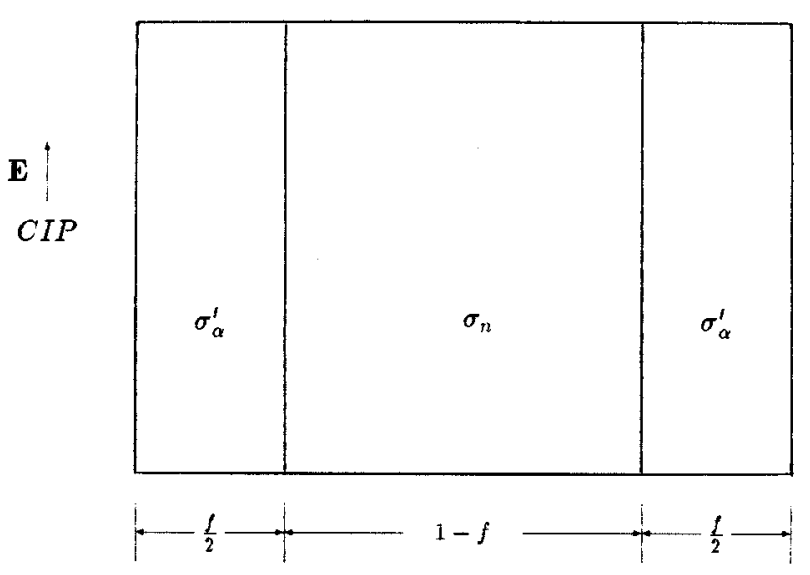

FIG. 1. Sandwich structure composed of two ferromagnetic films and one nonmagnetic film, with thickness proportional to $f$ and $1-f$, respectively.

CIP case of a multilayer. On the other hand, when $k_{\alpha}=\sigma_{n} / \sigma_{\alpha}^{\prime}$, Eqs. (9) and (10) become

$$
\begin{gathered}
\sigma^{M}=\left[f \sigma_{+}^{\prime-1}+(1-f) \sigma_{n}^{-1}\right]^{-1}+\left[f \sigma_{-}^{\prime-1}+(1-f) \sigma_{n}^{-1}\right]^{-1}, \\
\sigma^{D}=2\left[\frac{f}{2} \sigma_{+}^{\prime-1}+(1-f) \sigma_{n}^{-1}+\frac{f}{2} \sigma_{-}^{\prime-1}\right]^{-1} .
\end{gathered}
$$

They are just the expressions of resistivities of a resistor network in series, as shown in Fig. 1, and suitable for the CPP resistances of a magnetic multilayer in the local limit. Therefore, $k_{\alpha}=\sigma_{n} / \sigma_{\alpha}^{\prime}$ is equivalent to the CPP case of a multilayer.

\section{CALCULATION FOR $\boldsymbol{k}_{\boldsymbol{a}}$}

In this section we develop a macroscopic theory for granular systems to calculate $k_{\alpha}$. What we want to know is whether $k_{\alpha}$ is close to 1 (the CIP value) or close to $\sigma_{n} / \sigma_{\alpha}^{\prime}$ (the CPP value), or between these two values.

We first solve the problem of an isolated magnetic sphere embedded in a nonmagnetic metallic matrix (Fig. 2). In each

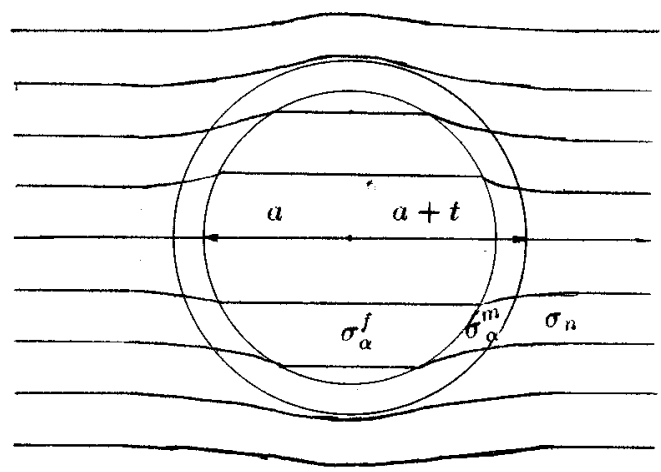

FIG. 2. Ferromagnetic sphere of radius $a$ and mixed sphere shell of thickness $t$. Lines of electric current are shown schematically. region the electric potentials acting on the spin-up and spindown conduction electrons are denoted as $\phi_{\alpha}$ (there is only one local magnetization direction in the whole system, so the relative spin sign $\alpha$ and the absolute spin sign $s$ have the same meaning).

In the absence of the spin-flip process, the potentials in each region satisfy the Laplace equations. ${ }^{18}$ Besides, there are six boundary conditions to be satisfied: one at infinity, one at the sphere's origin, and four at two interfaces of the sphere shell (mixed region). In the present model both the potential and the current must be continuous at the two interfaces. Using spherical coordinates, with the sphere centered at $\mathbf{r}=0$, and an electric field of magnitude $\mathbf{E}$ imposed parallel to the $z$ axis, the Laplace equations and the boundary conditions are

$$
\begin{gathered}
\nabla^{2} \phi_{\alpha}^{<}=0 \quad(r \leqslant a), \\
\nabla^{2} \phi_{\alpha}^{m}=0 \quad(a<r \leqslant a+t), \\
\nabla^{2} \phi_{\alpha}^{>}=0 \quad(r>a), \\
\phi_{\alpha}^{>} \rightarrow E r \cos \theta \quad(r \rightarrow \infty), \\
\phi_{\alpha}^{<}=\text {finity } \quad(r=0), \\
\phi_{\alpha}^{<}=\phi_{\alpha}^{m} \quad(r=a), \\
\phi_{\alpha}^{m}=\phi_{\alpha}^{>} \quad(r=a+t), \\
\sigma_{\alpha}^{f} \partial \phi_{\alpha}^{<} / \partial r=\sigma_{\alpha}^{m} \partial \phi_{\alpha}^{m} / \partial r \quad(r=a), \\
\sigma_{\alpha}^{m} \partial \phi_{\alpha}^{m} / \partial r=\sigma_{n} \partial \phi_{\alpha}^{>} / \partial r \quad(r=a+t),
\end{gathered}
$$

where $\phi_{\alpha}^{<}, \phi_{\alpha}^{m}$, and $\phi_{\alpha}^{>}$represent the spin-dependent potentials in the granule, mixed region, and matrix, respectively. The solutions of the Laplace equations are given by

$$
\begin{gathered}
\phi_{\alpha}^{<}=A_{\alpha} E r \cos \theta \quad(r \leqslant a), \\
\phi_{\alpha}^{m}=\left(B_{a}+a^{3} C_{\alpha} / r^{3}\right) E r \cos \theta \quad(a<r \leqslant a+t), \\
\phi_{\alpha}^{>}=\left[1+(a+t)^{3} D_{\alpha} / r^{3}\right] E r \cos \theta \quad(r>a+t),
\end{gathered}
$$

in which the spin-dependent coefficients $A_{\alpha}, B_{\alpha}, C_{\alpha}$, and $D_{\alpha}$ are determined by the boundary conditions of continuity as given above. A simple calculation yields

$$
\begin{gathered}
A_{\alpha}=9(a+t)^{3} \sigma_{\alpha}^{m} \sigma_{n} / F, \\
B_{\alpha}=3(a+t)^{3}\left(2 \sigma_{\alpha}^{m}+\sigma_{\alpha}^{f}\right) \sigma_{n} / F, \\
C_{\alpha}=3(a+t)^{3}\left(\sigma_{\alpha}^{m}-\sigma_{\alpha}^{f}\right) \sigma_{n} / F, \\
D_{\alpha}=\left[(a+t)^{3}\left(\sigma_{n}-\sigma_{\alpha}^{m}\right)\left(\sigma_{\alpha}^{f}+2 \sigma_{\alpha}^{m}\right)\right. \\
\left.-a^{3}\left(\sigma_{n}+2 \sigma_{\alpha}^{m}\right)\left(\sigma_{\alpha}^{f}-\sigma_{\alpha}^{m}\right)\right] / F,
\end{gathered}
$$

with

$$
\begin{aligned}
F= & (a+t)^{3}\left(2 \sigma_{n}+\sigma_{\alpha}^{m}\right)\left(2 \sigma_{\alpha}^{m}+\sigma_{\alpha}^{f}\right) \\
& +2 a^{3}\left(\sigma_{n}-\sigma_{\alpha}^{m}\right)\left(\sigma_{\alpha}^{m}-\sigma_{\alpha}^{f}\right) .
\end{aligned}
$$


From Eq. (12) and $\mathbf{E}_{\alpha}(\mathbf{r})=\nabla \phi_{\alpha}(\mathbf{r})$, the average fields in the sphere excluding and including the contribution of the mixed region are found to be

$$
\begin{gathered}
\overline{\mathbf{E}}_{\alpha}^{<}=A_{\alpha} \mathbf{E}, \\
\overline{\mathbf{E}^{\prime}}<=\left(1+D_{\alpha}\right) \mathbf{E} .
\end{gathered}
$$

The electric field in the matrix can be regarded as the sum of the external field $\mathbf{E}$ and the spin-dependent field produced by an effective electric dipole with dipole moment $(a+t)^{3} D_{\alpha} \mathbf{E}$ at the center of the sphere. ${ }^{18}$ When there are many spheres in the matrix with volumetric filling factor $f$, the mutual interaction between them should be taken into account. This effect can be calculated only to the lowest order by use of the Lorentz field approximation. Under this approximation the average field in the matrix is the external field plus a spindependent average field produced by all electric dipoles. Then the effective "external" field each magnetic granule feels is $\overline{\mathbf{E}}_{\alpha}^{>}$rather than $\mathbf{E}$, so that Eq. (14) should be corrected by replacing $\mathbf{E}$ with $\overline{\mathbf{E}}_{\alpha}^{>}$, that is

$$
\begin{gathered}
\overline{\mathbf{E}}_{\alpha}^{<}=A_{\alpha} \overline{\mathbf{E}}_{\alpha}^{>}, \\
\overline{\mathbf{E}^{\prime}}<=\left(1+D_{\alpha}\right) \overline{\mathbf{E}}_{\alpha}^{>} .
\end{gathered}
$$

In fact, we do not need to calculate $\overline{\mathbf{E}}_{\alpha}^{>}$, because what appears in Eqs. (9) and (10) for $\sigma^{M}$ and $\sigma^{D}$ is the ratio $k_{\alpha}^{G}$ $=\overline{\mathbf{E}^{\prime}}</ \overline{\mathbf{E}}_{\alpha}^{>}$and the effective conductivity $\sigma_{\alpha}^{\prime}=\bar{E}_{\alpha}^{<} \sigma_{\alpha}^{f} /{\overline{E^{\prime}}}_{\alpha}{ }_{\alpha}^{\alpha}$. From Eq. (15), they are given by

$$
k_{\alpha}^{G}=1+D_{\alpha}
$$

and

$$
\sigma_{\alpha}^{\prime}=\frac{A_{\alpha}}{1+D_{\alpha}} \sigma_{\alpha}^{f}
$$

Both the conductivity in the mixed region and its thickness are very small, but their ratio $r_{\alpha}=t / \sigma_{\alpha}^{m}$ has a finite value. Taking the limit $t \rightarrow 0$ in Eq. (13), we get

$$
\begin{gathered}
A_{\alpha}=\frac{3 a \sigma_{n}}{2 a \sigma_{n}+a \sigma_{\alpha}^{f}+2 r_{\alpha} \sigma_{n} \sigma_{\alpha}^{f}}, \\
D_{\alpha}=\frac{a \sigma_{n}-a \sigma_{\alpha}^{f}+r_{\alpha} \sigma_{n} \sigma_{\alpha}^{f}}{2 a \sigma_{n}+a \sigma_{\alpha}^{f}+2 r_{\alpha} \sigma_{n} \sigma_{\alpha}^{f}} .
\end{gathered}
$$

This limit means that the mixed region has been regarded as a sharp interface, and the defined $r_{\alpha}$ is an effective interfacial resistance per unit area. ${ }^{18}$ From Eqs. (16)-(18), we obtain $k_{\alpha}^{G}$ for a granular system

$$
k_{\alpha}^{G}=\frac{3 k_{\alpha}^{\mathrm{CPP}}}{2 k_{\alpha}^{\mathrm{CPP}}+1},
$$

with $k_{\alpha}^{\mathrm{CPP}}=\sigma_{n} / \sigma_{\alpha}^{\prime}$. It is straightforward to show from Eq. (19) that either $k_{\alpha}^{\mathrm{CPP}}>k_{\alpha}^{G}>1$ or $1>k_{\alpha}^{G}>k_{\alpha}^{\mathrm{CPP}}$, depending on $k_{\alpha}^{\mathrm{CPP}}>1$ or $k_{\alpha}^{\mathrm{CPP}}<1$. This indicates that $k_{\alpha}^{G}$ is always between $k_{\alpha}^{\mathrm{CIP}}=1$ and $k_{\alpha}^{\mathrm{CPP}}$. As has been discussed before, $k_{\alpha}$ is a parameter indicating the pattern of the resistor network:

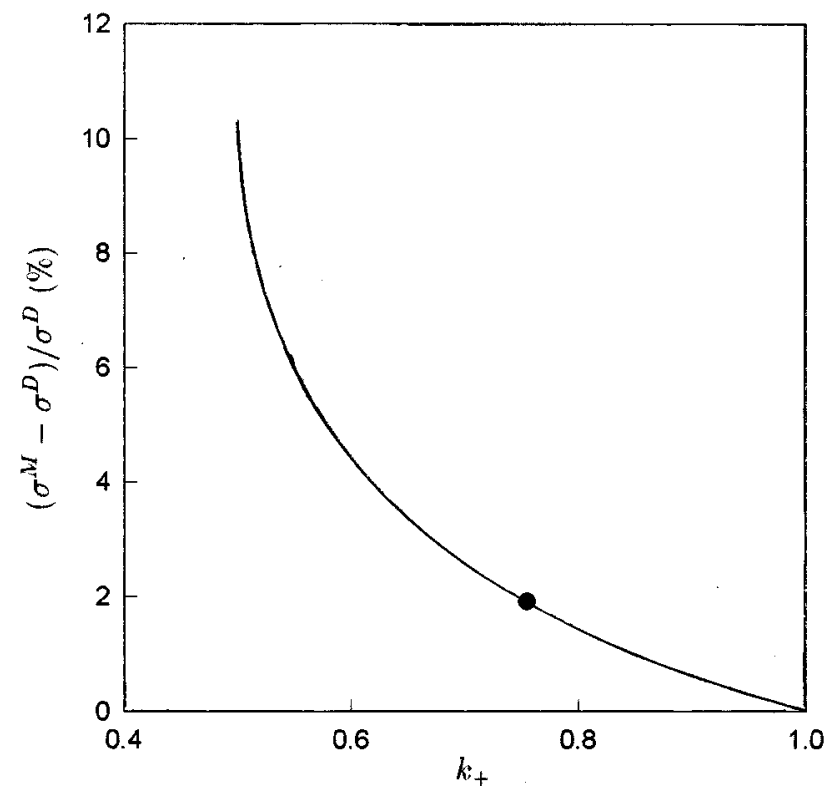

FIG. 3. Percent magnetoresistance as a function of $k_{+}$in a granular system. For $k_{+}^{\mathrm{CPP}}=1$ and $k_{+}^{\mathrm{CPP}}=0.5, k_{+}^{G}=0.75$ indicated as the solid circle.

$k_{\alpha}^{\mathrm{CIP}}=1$ for CIP and $k_{\alpha}^{\mathrm{CPP}}=\sigma_{n} / \sigma_{\alpha}^{\prime}$ for CPP. Thus it is concluded that the magnetotransport in a granular system is between those in the CIP and CPP case in a multilayer. From Eq. (19) we can see that if $k_{\alpha}^{\mathrm{CPP}}$ is very small, i.e., the conductivity of granules is much larger than that of the matrix, $k_{\alpha}^{G}$ is near $k_{\alpha}^{\mathrm{CPP}}$, and so the granular system is very close to the CPP case. Under the opposite condition in which the conductivity of granules is much smaller than that of the matrix, $k_{\alpha}^{G}$ is found very close to $k_{\alpha}^{\mathrm{CIP}}$, and the transport is similar to that in the CIP case. Although the present conclusion is derived in the local limit, it is expected to be applicable for more general scope. These results can be understood by the following physical interpretation. An important difference in transport between CPP and CIP is that the CPP currents must pass through the interface regions with high resistivity, while in the local limit the CIP currents may bypass these high resistivity regions. For a granular system there still exists the effect that the currents bypass the obstacles, especially in the case of $\sigma_{n}>\sigma_{\alpha}^{\prime}\left(k_{\alpha}^{\mathrm{CPP}}>1\right)$. It is this effect that leads to the transport of a granular system to be between the CPP and CIP cases. Considering the spindependent scattering in the granules and in the mixed regions, we assume the spin-asymmetry factors to be $\sigma_{+}^{f} / \sigma_{-}^{f}=3$ and $\sigma_{+}^{m} / \sigma_{-}^{m}=12$, the other parameters being $\sigma_{n}=\sigma_{-}$and $r_{-}=2 a / \sigma_{-}^{f}$ so that $k_{+}^{\mathrm{CPP}}=0.5 . k_{+}^{G}=0.75$ is shown as the solid circle in Fig. 3, from which one can find that when $k_{+}$varies from $k_{+}^{\mathrm{CPP}}$ to $k_{+}^{\mathrm{CIP}}=1$, the MR amplitude decreases monotonically. It is well known that in the local limit under consideration, the CIP-MR vanishes but the CPP-MR still exists due to the spin accumulation. The nonzero MR for $k_{+}^{G}$ in granular materials indicates that the spin accumulation still plays an important part in the MR, but has less effect than in the CPP geometry of the magnetic multilayers. 


\section{DISCUSSION}

We have developed a macroscopic theory to deal with the problem of GMR in granular magnetic metals in the case of $\lambda \ll d \ll l_{\text {sf }}$. At first glance, the present approach looks like that in Ref. 18. In reality, however, they are different from each other in several respects. First, in the present approach the average conductivity for each spin channel is defined as the average current density divided by the average electric field, as given in Eq. (5). The average field includes the internal field produced by spin accumulation, which is closely related to the actual potential drop on the granular sample. In Ref. 18, the external field rather than the average field was used in the definition of the average conductivity. Such an improper treatment would result in a positive MR, which is undoubtedly an unphysical result. Second, in the Lorentz field approximation the field produced by all effective dipoles is roughly treated as a uniform background field. In the present approach this background field plus the external field is regarded as an effective "external" field and the potentials $\phi_{\alpha}^{<}, \phi_{\alpha}^{m}$, and $\phi_{\alpha}^{>}$are calculated self-consistently as done in Sec. III. In Ref. 18, however, the Lorentz background field is simply added to the field inside the ferromagnetic granules as well as in the matrix. Third, only one spin channel is considered in Ref. 18 and both spin channels are taken into account in our work. Since the conductivities for both spin channels in the matrix are the same, the minorityspin channel always has contribution to the total conductivity and so cannot be neglected, even if the conductivity for the minority-spin electrons is zero inside the spheres, as has been assumed in Ref. 18.

Finally, we use the present macroscopic theory to calculate the GMR varying with the granule size. All the parameters are taken to be the same values as used in Sec. III (Fig. 3 ) and are assumed to remain independent of granule size. In Fig. 4 we plot the MR amplitude as a function of the sphere radius $a$. From Fig. 4 we find that the MR first increases and then decreases with increasing the granule size, and a maximum occurs in the middle region. As seen from the experimental observations, ${ }^{5}$ there is always an optimum annealing temperature in preparing the magnetic granular sample for GMR. In view of the fact that the sizes of the particles are always increased with increasing the annealing temperature, our results are in good agreement with these observations. Here we wish to make a simple physical interpretation of our calculated results. The behavior that the MR increases with decreasing $a$ is easily understood by the argument ${ }^{11}$ that with decreasing $a$, the ratio of interface area to the granule volume always increases, and so the spin-dependent interface scattering responsible to the GMR becomes strong. On the other hand, when $a$ is small enough there appears an opposite tendency that the MR decreases with further decreasing $a$. For small granules the effect of interface scattering is much larger than that of the bulk scattering, making the effective conductivity of granules much smaller than that of the matrix. As has been discussed in Sec. III, in this case $k_{\alpha}^{G}$ moves towards $k_{\alpha}^{\mathrm{CIP}}$ so that the magnetotransport of the granular system is close to the CIP case in multilayers and the MR decreases rapidly with further decreasing $a$. Therefore, such an opposite size dependence of the MR is closely related to a crossover of the magnetotransport from CPP to

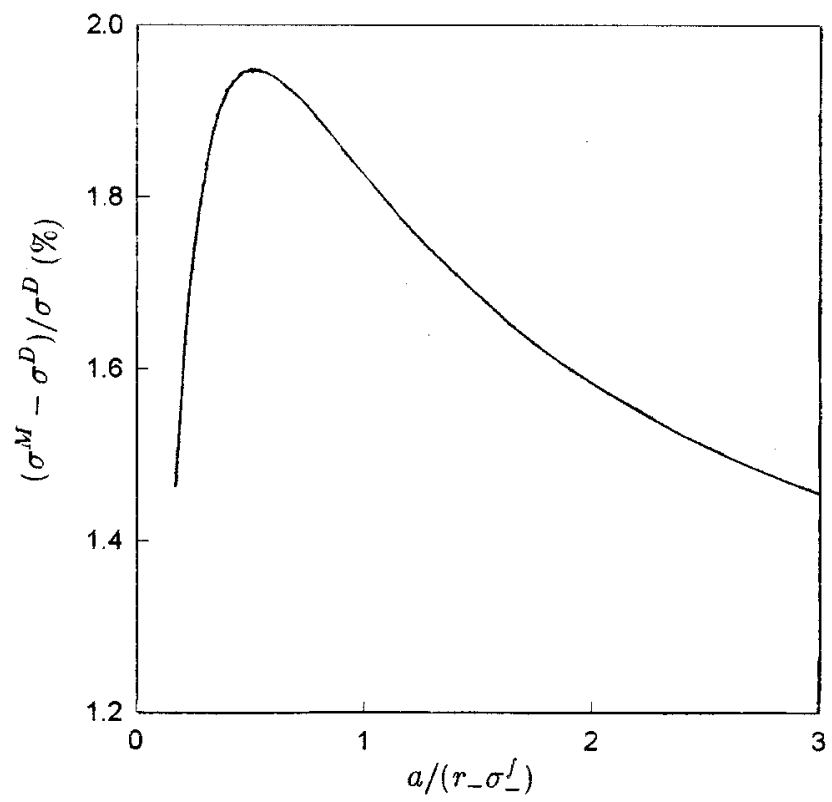

FIG. 4. Percent magnetoresistance as a function of the size of the granules $a$ for a granular sample composed of ferromagnetic spheres of radius $a$ packed with filling factor $f$. It is assumed the conductivities do not vary with $a$.

CIP, and it cannot be obtained by using a pure CPP picture at very low temperatures. Although the calculated result shown in Fig. 4 is obtained in the local limit and the further work in the nonlocal case is highly required, the above argument on the size dependence of the GMR is believed to be universal for arbitrary length scales. It is worth mentioning that for magnetic granular systems a size dependence of the MR similar to that shown in Fig. 4 was obtained by Zhang and Levy $^{17}$ in terms of a different mechanism. In their theory the increase in MR with the size of the granules was attributed to the consideration that for a fixed magnetic field and temperature, very small granules cannot be saturated due to the superferromagnetic effect.

In summary, we have developed a macroscopic theory of GMR in magnetic granular metals. Taking both spin channels into account and using a self-consistent way in dealing with the internal electric field, the conductivities of a granular system in both the magnetized state and the demagnetized state have been studied. By introducing a useful parametrization, we find that in the local limit the magnetotransport in magnetic granular systems is between those for CPP and CIP in magnetic multilayers. The calculated result shows that with decreasing the size of the ferromagnetic granules, the MR first increases and then decreases, exhibiting a maximum in the middle region, which is in good agreement with the size dependence of the GMR observed experimentally in magnetic granular metals.

\section{ACKNOWLEDGMENTS}

This work was supported by the Natural Science Foundation and the National Doctoral Education Foundation in China, and in part by a joint grant of the NSFC and NAMCC of China. 
${ }^{1}$ M. N. Baibich, J. M. Broto, A. Fert, Van. Dau. F. Nguygen, F. Petroff, P. Eitenne, G. Creuzet, A. Friedrich, and J. Chazelas, Phys. Rev. Lett. 61, 2472 (1988).

${ }^{2}$ S. S. P. Parkin, N. More, and K. P. Roche, Phys. Rev. Lett. 64, 2304 (1990).

${ }^{3}$ Jr. W. P. Pratt, S-F. Lee, J. M. Slaughter, R. Loloee, P. A. Schroder, and J. Bass, Phys. Rev. Lett. 66, 3060 (1991).

${ }^{4}$ M. A. M. Gijs, S. K. J. Lenczowski, and J. B. Giesbers, Phys. Rev. Lett. 70, 3343 (1993).

${ }^{5}$ A. E. Berkowitz et al., Phys. Rev. Lett. 68, 3745 (1992); J. Q. Xiao, J. S. Jiang, and C. L. Chien, ibid. 68, 3749 (1992).

${ }^{6}$ J. Q. Xiao, J. S. Jiang, and C. L. Chien, Phys. Rev. B 46, 9266 (1992).

${ }^{7}$ R. E. Camley and J. Barnas, Phys. Rev. Lett. 63, 664 (1989).

${ }^{8}$ B. L. Johnson and R. E. Camley, Phys. Rev. B 44, 9997 (1991).

${ }^{9}$ R. Q. Hood and L. M. Falicov, Phys. Rev. B 46, 8287 (1992).
${ }^{10}$ M. Liu and D. Y. Xing, Phys. Rev. B 47, 12272 (1993); L. Sheng and D. Y. Xing, ibid. 50, 1001 (1994).

${ }^{11}$ P. M. Levy, S. Zhang, and A. Fert, Phys. Rev. Lett. 65, 1643 (1990).

${ }^{12}$ A. Vedyayev, B. Dieny, and N. Ryzhanova, Europhys. Lett. 19, 329 (1992).

${ }^{13}$ M. Johnson, Phys. Rev. Lett. 67, 3594 (1991).

${ }^{14}$ T. Valet and A. Fert, Phys. Rev. B 48, 7099 (1993).

${ }^{15}$ H. E. Camblong, S. Zhang, and P. M. Levy, Phys. Rev. B 47, 4735 (1993).

${ }^{16}$ S. Zhang, Appl. Phys. Lett. 61, 1855 (1992).

${ }^{17}$ S. Zhang and P. M. Levy, J. Appl. Phys. 73, 5315 (1993).

${ }^{18}$ M. Rubinstein, Phys. Rev. B 50, 3830 (1994).

${ }^{19}$ P. B. Zhao and F. C. Pu, Phys. Rev. B 51, 11603 (1995).

${ }^{20}$ H. E. Camblong, P. M. Levy, and S. Zhang, Phys. Rev. B 51, 16052 (1995). 